\title{
Is there a role for immunotherapy in ovarian cancer?
}

\author{
Gaia Giannone $^{1,2}$, Giorgio Valabrega ${ }^{1,2}$ \\ ${ }^{1}$ FPO-IRCCS Candiolo, Candiolo Cancer Institute, Candiolo, Italy; ${ }^{2}$ Department of Oncology, University of Torino, Torino, Italy \\ Correspondence to: Dr. Giorgio Valabrega. Candiolo Cancer Institute, FPO - IRCCS - Str. Prov.le 142, km. 3,95 - Candiolo (TO) 10060, Italy; \\ Department of Oncology, University of Torino, Torino, Italy. Email: giorgio.valabrega@ircc.it. \\ Provenance: This is an invited article commissioned by the Editorial Office, Annals of Translational Medicine. \\ Comment on: Matulonis UA, Shapira-Frommer R, Santin AD, et al. Antitumor activity and safety of pembrolizumab in patients with advanced \\ recurrent ovarian cancer: results from the phase II KEYNOTE-100 study. Ann Oncol 2019;30:1080-7.
}

Submitted Nov 01, 2019. Accepted for publication Nov 25, 2019.

doi: $10.21037 / \mathrm{atm} .2019 .12 .13$

View this article at: http://dx.doi.org/10.21037/atm.2019.12.13

Epithelial ovarian cancer (OC) is the most lethal gynaecological malignancy worldwide with 22,530 estimated new cases in 2019, 13,980 deaths in 2019 and a median overall survival (OS) of less than 5 years (1). Indeed most of the cases are diagnosed in advanced stage and despite optimal management with a combination of surgery and platinum-base chemotherapy, most patients recur (2). The introduction of PARP inhibitors in treatment algorithms, has changed prognosis above all of BRCA mutated and homologous recombination deficient (HRD) positive patients but recurrence is still associated with development of drug resistance (and above all platinum resistance), low response rate to subsequent therapies and eventually death (2).

Among different therapeutic strategies one of the most appealing seems to be the use of immune modulating agents. Preclinical data suggest that immune microenvironment has a prognostic role also in OC. Presence of a high number of intratumor infiltrating lymphocytes (TILs) has been associated with a longer OS $(3,4)$ and although OC has a low tumor mutational burden (TMB), it is well known that HRD positive patients, having an impaired mechanism of DNA repair, express a higher percentage of neoantigens (5). Indeed patients with both HRD and high $\mathrm{CD} 3+\mathrm{T}$ lymphocytes have a longer OS if compared to HR proficient patients with low CD3+ T lymphocytes (6). On the other hand data on PDL-1 expression seems to be uncertain, indeed works described it both as a positive $(7,8)$ and negative (9) prognostic factor.

In a context of limited therapeutic options for relapsed disease, several early phase studies evaluated the role of immune checkpoint inhibitors in OC patients. In
Keynote-100 (10), a large II trial, 376 patients with recurrent $\mathrm{OC}$ have been treated with Pembrolizumab $200 \mathrm{mg}$ administered intravenously every 3 weeks. The study population was divided in two cohorts of patients: cohort $\mathrm{A}$, including patients that received more than 1 and less than 3 prior lines with a platinum free interval (PFI) or treatment free interval (TF) (that is the time elapsed from last platinum-based cycle or last treatment respectively and evidence of disease progression) of 3-12 months and cohort $\mathrm{B}$ receiving four to six prior lines with a PFI or TFI longer than 3 months. Primary endpoint was overall response rate (ORR) per RECIST1.1 by blinded independent central review (BICR) in cohort A and B and by PDL-1 expression as described below (10).

The ORR in the overall population was $8.0 \%$ with $7.4 \%$ in cohort A, 9.9\% in cohort B. Disease control rate (DCR) was about $37 \%$. Median progression free survival (PFS) was 2.1 months with a median OS not reached in cohort A and 17.6 months in cohort B (10).

PDL-1 expression was defined with the PD-L1 IHC 22C3 pharmDx assay, Dako North America using a combined positive score (CPS) that is "the number of PDL1 staining cells (tumor cells, lymphocytes, macrophages) divided by the total number of viable tumor cells" (10). In patients with CPS $<1$ ORR was $5.0 \%$ whereas it was $10.2 \%$ for CPS $\geq 1$, and $17.1 \%$ for CPS $\geq 10$ patients. No difference in HRD and BRCA status was shown between responders and nonresponders (10).

Results from Keynote-100 confirm the activity observed in Keynote-028, where patients with platinum resistant OC and PDL1 positivity, defined as PDL-1 expression in more 
than $1 \%$ of cells in tumor, achieved an ORR of $11.5 \%$ with pembrolizumab (11). Moreover, these data are comparable to those from other early phase trials testing other immune checkpoint inhibitors like avelumab, nivolumab and atezolizumab in EOC (12-14).

Keynote-100 (10) is the largest published study with an anti-PD1 in OC patients. Interestingly, its primary endpoint was not only to evaluate activity of pembrolizumab but to define a cutoff in PDL-1 expression that could discriminate responders from non-responders. Apparently, also this endpoint was met, being outcome in PDL-1 better than patients with PDL1 low.

Nevertheless several question are still open. First of all, defined cutoff is highly dependent on the type of test, indeed studies in other cancers suggests that assays are not interchangeable and that they could miss some PDL1 high patients (15). Moreover role of PDL-1 depends on the type of cells with PDL-1 expression (cancer cells or immune cells) and there is a high temporal and spatial heterogeneity (16). Second, we cannot define if PDL-1 high is only prognostic or also predictive of a higher response specifically to immunotherapy. In other malignancies like non-small cell lung cancers (NSCLC), prognostic role of PDL-1 is not defined $(17,18)$, but it is a good predictive biomarker both for pembrolizumab alone or in combination with chemotherapy in first line treatment (19-21). Also in cisplatin- ineligible patients with advanced urothelial cancer, a high expression of PDL-1 correlated with a better response to pembrolizumab (22). This led to FDA approval of pembrolizumab single agent in both malignancies only in patients with a high expression of PDL-1 (23).

Several other biomarkers could integrate PDL-1. Among these Mismatch repair deficiency (24), TMB that are indirect markers of high neoepitope production or $\mathrm{T}$ cellinflamed gene expression profile (GEP) that indicate an "inflamed" tumor microenvironment (25).

Once again, also combined biomarkers don't fit with OC. A low percentage of OC patients has a high TMB and T-cell GEP or shows MMR deficiency $(24,25)$, features that seem to predict a higher ORR and a longer OS with checkpoint inhibitors.

Therefore, even if Keynote-100 suggests that PDL-1 could be a good predictive biomarker, ORR in patients with CPS $>10$ is comparable with single agent chemotherapy (2) and does not help in identifying subgroups of patients that might long term benefit from the use of pembrolizumab.

The core question is if we are missing the point. Is the problem the absence of a predictive biomarker or that this therapeutic strategy is not successful in OC patients. Indeed, in other malignancies, like melanoma, although a defined biomarker is still unavailable, results from several studies with long follow up suggest that immune checkpoint inhibitors guarantee a high response rate with a flat plateau of long responders at five years (26).

Data from early phase trials in OC patients, on the other hand, suggest that PD1/PD-L1 blockade is not enough powerful to achieve a clinically relevant response but several trials are evaluating different strategies to overcome immune tolerance.

An interesting approach is to combine immune checkpoint inhibitors with other agents that could enhance response. Among these, the most promising partners for immunotherapy seem to be PARP inhibitors (27). Indeed results from MEDIOLA study describe an ORR of more than $70 \%$ in platinum sensitive gBRCA mutated OC patients treated with durvalumab and olaparib, suggesting that probably both a selection of patients and a combination of treatments could improve outcome (27). Nevertheless patients with platinum resistant OC in TOPACIO study achieved an ORR of $18 \%$ with a combination of niraparib and pembrolizumab, results comparable to other standard treatment in this setting (27).

Other possible strategies under investigation to exploit tumor microenvironment are adoptive immune therapies (28). Indeed infusion of autologous or allogenic immune cells (for example TILs or engineered cells like CART) show promising results in preclinical settings and data from a phase I trial demonstrate that OC patients treated with TILs after surgery and cisplatin based therapy have an OS of $100 \%$ compared to $67.5 \%$ in patients treated with chemotherapy only (27).

Although results are preliminary and further data are needed, they give hope that also patients with OC could benefit from immunotherapy. In this context translational research is crucial to better understand immune system and successfully improve immune response against OC.

\section{Acknowledgments}

This work was funded by Ministero della Salute, Ricerca Corrente 2019.

\section{Footnote}

Conflicts of Interest: G Valabrega has received personal fees from Roche, AstraZeneca, Tesaro, PharmaMar, Amgen. G 
Giannone has no conflicts of interest to declare.

Ethical Statement: The authors are accountable for all aspects of the work in ensuring that questions related to the accuracy or integrity of any part of the work are appropriately investigated and resolved.

\section{References}

1. Available online: https://seer.cancer.gov/statfacts/html/ ovary.html, 2019.

2. Colombo N, Sessa C, du Bois A, et al. ESMO-ESGO consensus conference recommendations on ovarian cancer: pathology and molecular biology, early and advanced stages, borderline tumours and recurrent disease. Ann Oncol 2019;30:672-705.

3. Zhang L, Conejo-Garcia JR, Katsaros D, et al. Intratumoral $\mathrm{T}$ cells, recurrence, and survival in epithelial ovarian cancer. N Engl J Med 2003;348:203-13.

4. Hwang WT, Adams SF, Tahirovic E, et al. Prognostic significance of tumor-infiltrating $\mathrm{T}$ cells in ovarian cancer: a meta-analysis. Gynecol Oncol 2012;124:192-8.

5. Strickland KC, Howitt BE, Shukla SA, et al. Association and prognostic significance of BRCA1/2-mutation status with neoantigen load, number of tumor-infiltrating lymphocytes and expression of PD-1/PD-L1 in high grade serous ovarian cancer. Oncotarget 2016;7:13587-98.

6. Morse CB, Toukatly MN, Kilgore MR, et al. Tumor infiltrating lymphocytes and homologous recombination deficiency are independently associated with improved survival in ovarian carcinoma. Gynecol Oncol 2019;153:217-22.

7. Webb JR, Milne K, Kroeger DR, et al. PD-L1 expression is associated with tumor-infiltrating $\mathrm{T}$ cells and favorable prognosis in high-grade serous ovarian cancer. Gynecol Oncol 2016;141:293-302.

8. Darb-Esfahani S, Kunze CA, Kulbe H, et al. Prognostic impact of programmed cell death-1 (PD-1) and PD-ligand 1 (PD-L1) expression in cancer cells and tumor-infiltrating lymphocytes in ovarian high grade serous carcinoma. Oncotarget 2016;7:1486-99.

9. Abiko K, Matsumura N, Hamanishi J, et al. IFN- $\gamma$ from lymphocytes induces PD-L1 expression and promotes progression of ovarian cancer. $\mathrm{Br} \mathrm{J}$ Cancer 2015;112:1501-9.

10. Matulonis UA, Shapira-Frommer R, Santin AD, et al. Antitumor activity and safety of pembrolizumab in patients with advanced recurrent ovarian cancer: results from the phase II KEYNOTE-100 study. Ann Oncol 2019;30:1080-7.

11. Varga A, Piha-Paul SA, Ott PA, et al. Antitumor activity and safety of pembrolizumab in patients (pts) with PD-L1 positive advanced ovarian cancer: Interim results from a phase Ib study. J Clin Oncol 2015;33; abstr 5510.

12. Disis ML, Patel MR, Pant $S$, et al. Avelumab (MSB0010718C; anti-PD-L1) in patients with recurrent/ refractory ovarian cancer from the JAVELIN Solid Tumor phase Ib trial: Safety and clinical activity. J Clin Oncol 2016;34:abstr 5533.

13. Hamanishi J, Mandai M, Ikeda T, et al. Safety and Antitumor Activity of Anti-PD-1 Antibody, Nivolumab, in Patients With Platinum-Resistant Ovarian Cancer. J Clin Oncol 2015;33:4015-22.

14. Liu JF, Gordon M, Veneris J, et al. Safety, clinical activity and biomarker assessments of atezolizumab from a Phase I study in advanced/recurrent ovarian and uterine cancers. Gynecol Oncol 2019;154:314-22.

15. Hirsch FR, McElhinny A, Stanforth D, et al. PDL1 Immunohistochemistry Assays for Lung Cancer: Results from Phase 1 of the Blueprint PD-L1 IHC Assay Comparison Project. J Thorac Oncol 2017;12:208-22.

16. Ung C, Kockx MM. Challenges \& Perspectives of Immunotherapy Biomarkers \& The HistoOncoImmune ${ }^{\mathrm{TM}}$ Methodology. Expert Rev Precis Med Drug Dev 2016;1:9-24.

17. Cooper WA, Tran T, Vilain RE, et al. PD-L1 expression is a favorable prognostic factor in early stage non-small cell carcinoma. Lung Cancer 2015;89:181-8.

18. Wang A, Wang HY, Liu Y, et al. The prognostic value of PD-L1 expression for non-small cell lung cancer patients: a meta-analysis. Eur J Surg Oncol 2015;41:450-6.

19. Langer CJ, Gadgeel SM, Borghaei H, et al. Carboplatin and pemetrexed with or without pembrolizumab for advanced, non-squamous non-small-cell lung cancer: a randomised, phase 2 cohort of the open-label KEYNOTE-021 study. Lancet Oncol 2016;17:1497-508.

20. Reck M, Rodríguez-Abreu D, Robinson AG, et al. Pembrolizumab versus Chemotherapy for PD-L1Positive Non-Small-Cell Lung Cancer. N Engl J Med 2016;375:1823-33.

21. Leighl NB, Hellmann MD, Hui R, et al. Pembrolizumab in patients with advanced non-small-cell lung cancer (KEYNOTE-001): 3-year results from an open-label, phase 1 study. Lancet Respir Med 2019;7:347-57.

22. Balar AV, Castellano D, O'Donnell PH, et al. First-line pembrolizumab in cisplatin-ineligible patients with locally 
advanced and unresectable or metastatic urothelial cancer (KEYNOTE-052): a multicentre, single-arm, phase 2 study. Lancet Oncol 2017;18:1483-92.

23. fda.gov. Available online: https://www.accessdata.fda.gov/ drugsatfda_docs/label/2019/125514s065lbl.pdf, 2019.

24. Le DT, Durham JN, Smith KN, et al. Mismatch repair deficiency predicts response of solid tumors to PD-1 blockade. Science 2017;357:409-13.

25. Cristescu R, Mogg R, Ayers M, et al. Pan-tumor genomic biomarkers for PD-1 checkpoint blockade-based

Cite this article as: Giannone G, Valabrega G. Is there a role for immunotherapy in ovarian cancer? Ann Transl Med 2019;7(Suppl 8):S276. doi: 10.21037/atm.2019.12.13 immunotherapy. Science 2018;362:6411.

26. Larkin J, Chiarion-Sileni V, Gonzalez R, et al. Five-Year Survival with Combined Nivolumab and Ipilimumab in Advanced Melanoma. N Engl J Med 2019;381:1535-46.

27. Ghisoni E, Imbimbo M, Zimmermann S, et al. Ovarian Cancer Immunotherapy: Turning up the Heat. Int J Mol Sci 2019;20:12.

28. Mittica G, Capellero S, Genta S, et al. Adoptive immunotherapy against ovarian cancer. J Ovarian Res 2016;9:30. 